\begin{tabular}{|c|l|}
\hline Title & Reentrant transition in coupled noisy oscillators \\
\hline Author(s) & Kobay ashi, Y asuaki; Kori, Hiroshi \\
\hline Citation & $\begin{array}{l}\text { Physical review. E, 91(1), 12901-1-12901-6 } \\
\text { https://doi.org/10.1103/PhysRevE.91.012901 }\end{array}$ \\
\hline Issue Date & 2015-01-05 \\
\hline Doc URL & http://hdl.handle.net/2115/58135 \\
\hline Rights & @2015A merican Physical Society \\
\hline Type & article \\
\hline File Information & PhysRevE.91.012901.pdf \\
\hline
\end{tabular}

Instructions for use 


\title{
Reentrant transition in coupled noisy oscillators
}

\author{
Yasuaki Kobayashi* \\ Research Institute for Electronic Science, Hokkaido University, Sapporo 060-0812, Japan \\ Hiroshi Kori \\ Department of Information Sciences, Ochanomizu University, Tokyo 112-8610, Japan
}

(Received 6 October 2014; revised manuscript received 9 December 2014; published 5 January 2015)

\begin{abstract}
We report on a synchronization-breaking instability observed in a noisy oscillator unidirectionally coupled to a pacemaker. Using a phase oscillator model, we find that, as the coupling strength is increased, the noisy oscillator lags behind the pacemaker more frequently and the phase slip rate increases, which may not be observed in averaged phase models such as the Kuramoto model. Investigation of the corresponding Fokker-Planck equation enables us to obtain the reentrant transition line between the synchronized state and the phase slip state. We verify our theory using the Brusselator model, suggesting that this reentrant transition can be found in a wide range of limit cycle oscillators.
\end{abstract}

DOI: 10.1103/PhysRevE.91.012901

PACS number(s): 05.45.Xt, 05.40.-a

\section{INTRODUCTION}

Synchronization of self-sustained oscillators [1-3] is crucial in many systems, including cardiac cells [4,5], circadian clock cells [6,7], and power grids [8-10]. These systems are inevitably subject to various kinds of perturbations such as inherent noise, inhomogeneity, and environmental changes, and therefore coupling of such oscillators needs to be strong enough to overcome the effect of these disturbances and ensure synchronization.

It is known that strong coupling can be a source of instabilities including oscillation death [11,12] and chaotic dynamics $[13,14]$. However, many of previous studies on coupled oscillators in the presence of noise focus on the competition of coupling and noise [3,15-17], where coupling is expected to suppress noise, leading to fast and stable synchronization.

In this paper, we present a synchronization-breaking scenario which can occur in the strong coupling regime. Naturally, synchronization does not occur for too weak coupling because of the effect of noise. We find that, in addition to this trivial desynchronization, synchronization is disrupted also for too strong coupling. Such a reentrant transition occurs even with very weak noise. We elucidate the condition and mechanism of this reentrant synchronization through the analysis of a phase oscillator model. Furthermore, we verify that the same reentrant transition occurs in limit-cycle oscillators by using the Brusselator model and confirm the validity of our theory. Our study demonstrates that the reentrant transition appears quite generally in coupled noisy oscillators.

\section{COUPLED PHASE OSCILLATOR MODEL UNDER NOISE}

We consider the following phase oscillator that is subject to noise and is influenced by a noise-free pacemaker:

$$
\dot{\phi}=\omega+K Z(\phi)\{h(\Omega t)-h(\phi)\}+\xi(t),
$$

\footnotetext{
*yasuaki.kobayashi@es.hokudai.ac.jp
}

where $\phi$ is the phase, $\omega$ and $\Omega$ are the frequency of the oscillator and the pacemaker, respectively, and $\xi(t)$ is a Gaussian white noise satisfying $\left\langle\xi(t) \xi\left(t^{\prime}\right)\right\rangle=D \delta\left(t-t^{\prime}\right)$. Interaction is determined by $2 \pi$-periodic functions $Z(\phi)$ and $h(\phi)$. A large class of limit-cycle oscillator models can be reduced to this model when the stability of a limit-cycle oscillator is high enough compared to noise and coupling strengths [2]. Here we adopt the following simple functions:

$$
Z(\phi)=\sin (\phi-\alpha), \quad h(\phi)=-\cos \phi,
$$

with a parameter $\alpha$. Below we mostly consider the case $\Omega=\omega$.

\section{A. Averaged model}

Let us first examine the averaged dynamics. When the coupling strength $K(>0)$ and the noise strength $D$ are sufficiently small compared to $\omega$, Eq. (1) is well approximated by an averaged phase model. When $\Omega=\omega$, the phase difference $\psi \equiv \phi-\omega t$ obeys

$$
\dot{\psi}=K \Gamma(\psi)+\xi(t)
$$

where the interaction function $\Gamma$ is obtained as [2]

$$
\Gamma(\psi) \equiv \frac{1}{2 \pi} \int_{0}^{2 \pi} d \theta Z(\psi+\theta)\{h(\theta)-h(\psi+\theta)\} .
$$

The present choice of $Z$ and $h$ yields a Sakaguchi-Kuramoto type interaction function [18],

$$
\Gamma(\psi)=-\frac{1}{2}\{\sin (\psi-\alpha)+\sin \alpha\} .
$$

In the absence of noise, the state $\psi=0$ is stable when $\Gamma^{\prime}(0)=$ $-\frac{1}{2} \cos \alpha$ is negative, i.e., $-\pi / 2<\alpha<\pi / 2$, which we always consider in the present paper.

It is convenient to rewrite Eq. (3) as

$$
\dot{\psi}=-K \frac{\partial F(\psi)}{\partial \psi}+\xi(t)
$$



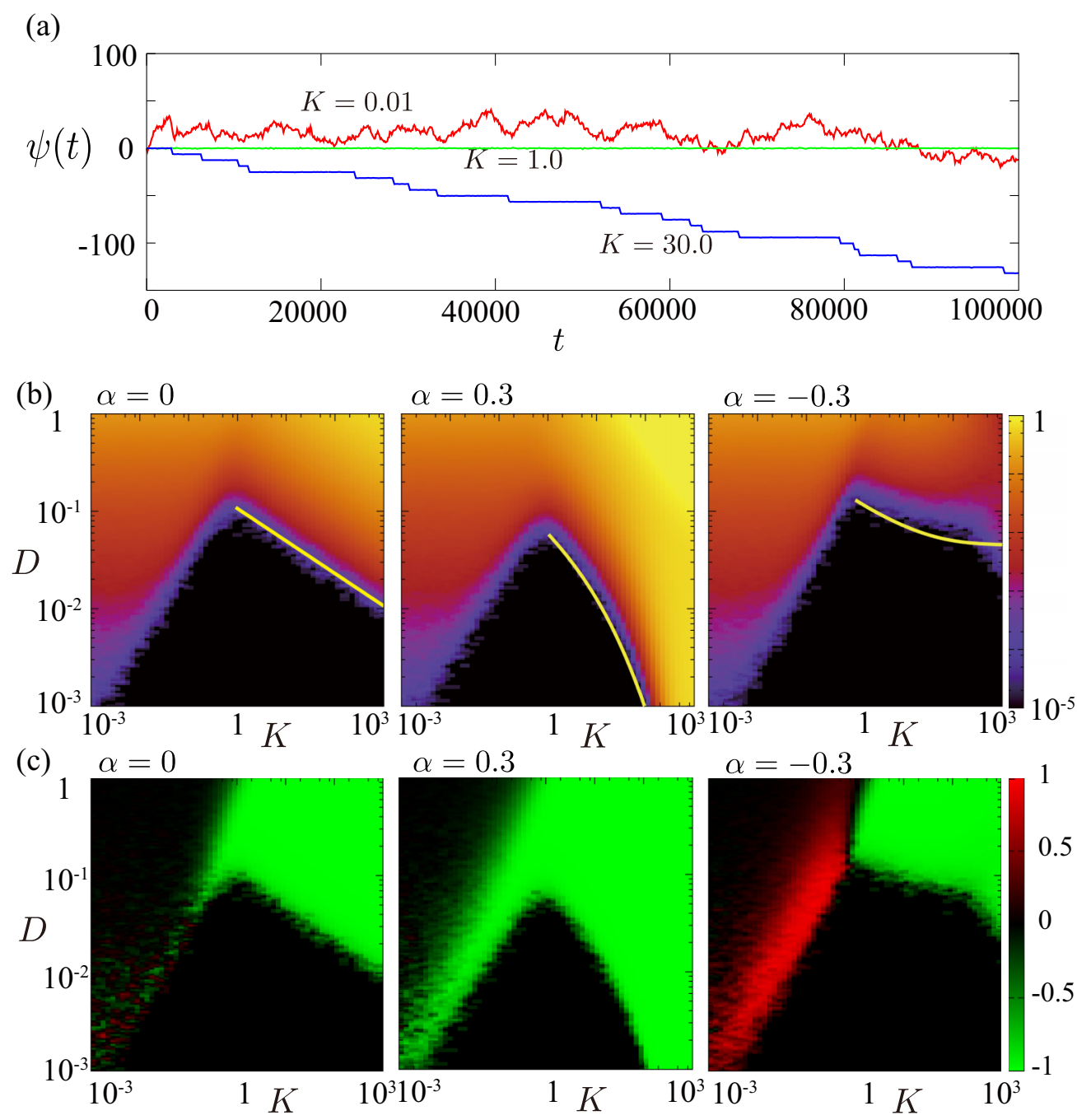

FIG. 1. (Color online) (a) Time evolution of the phase difference $\psi$ for three different values of $K$, with $\alpha=0$ and $D=0.05$. (b) Phase slip rate against the coupling strength $K$ and the noise intensity $D$ for $\alpha=0,0.3$, and -0.3 . Theoretical lines are given by Eqs. (18) and (19) for $K \geqslant 1$. (c) Unidirectionality of slip against $K$ and $D$. Red (appearing only in the region $K<1$ for $\alpha=-0.3$ ) and green indicate positive and negative directions of $\psi$, respectively.

where the potential $F$ is given by

$$
\begin{aligned}
F(\psi) & \equiv-\int_{0}^{\psi} d \psi^{\prime} \Gamma\left(\psi^{\prime}\right) \\
& =-\frac{1}{2} \cos (\psi-\alpha)+\frac{1}{2}(\sin \alpha) \psi .
\end{aligned}
$$

If $K \gtrsim D$, the phase difference $\psi$ tends to stay around the potential minima, and occasionally jumps to the two adjacent minima, driven by noise. If $\alpha=0$, the right and the left potential barriers are the same height, and no net drift appears. On the other hand, if $\alpha \neq 0$, the imbalance of the two barriers causes nonzero drift in the positive direction for $\alpha<0$, or in the negative direction for $\alpha>0$. The rate of phase slip is well approximated by Kramers' formula [3]: Given a barrier height $\Delta F$, the rate of overcoming the barrier is proportional to $\exp \left(-\frac{K \Delta F}{D}\right)$. Since the difference between the right and the left barriers is $\pi \sin \alpha$, the net jump rate is proportional to

$$
\exp \left(-\frac{\Delta F_{\mathrm{L}} K}{D}\right)\left\{\exp \left(-\frac{K \pi \sin \alpha}{D}\right)-1\right\},
$$

where $\Delta F_{\mathrm{L}}$ is the height of the left barrier. That is, in the averaged dynamics, the rate of phase slip decreases exponentially as $K$ increases, with its direction determined by the sign of $\alpha$, and no reentrant transition occurs.

\section{B. Nonaveraged model}

When $K$ or $D$ is not sufficiently smaller than $\omega$, the averaging is no longer valid. In this case, the situation changes drastically. To see this, we numerically solve Eq. (1). The frequency $\omega$ can be set to unity without loss of generality. Figure 1(a) shows three types of behavior for different values of $K$ with $D=0.05$ and $\alpha=0$ : For $K=0.01$, the system is dominated by noise (incoherent region). Synchronization is observed when $K$ is increased to $K=1.0$. However, as we further increase $K$ up to $K=30$, synchronization is disturbed by a jump of the phase difference by $-2 \pi$ (i.e., a phase slip). Measuring the rate of phase slip events against $K$ and $D$ for different values of $\alpha$, we observe the reentrant transition from 
the synchronized state to the phase slip state [Fig. 1(b)]. The critical value of $K$ for this transition decreases as $D$ increases.

Unidirectionality of the slips is seen in Fig. 1(c). Here, for each $K$ and $D$, we plot $\left(N_{+}-N_{-}\right) /\left(N_{+}+N_{-}+\epsilon\right)$, where $N_{+}$ and $N_{-}$are the number of slips in the positive and the negative directions, respectively, and $\epsilon=1$ is inserted to circumvent zero division. In the incoherent region, the slip direction and frequency is determined by the sign of $\alpha$, reflecting the drifting force $-\frac{K}{2} \sin \alpha$ appearing in the averaged dynamics. On the other hand, in the reentrant region, the slip direction is negative for all $\alpha$ values, implying that the phase slip in the nonaveraged dynamics is qualitatively different from that in the averaged dynamics.

The local stability analysis cannot explain the reentrant transition. Linearizing Eq. (1) around the synchronized state $\psi=0$ with $\omega=\Omega$ yields

$$
\dot{\psi}=-K \lambda(\omega t) \psi+\xi(t)
$$

where $\lambda(\omega t) \equiv Z(\omega t) h^{\prime}(\omega t)$. The linear stability of the state $\psi=0$ is determined by the time average of $\lambda(\omega t)$, i.e., $(\omega / 2 \pi) \int_{0}^{2 \pi / \omega} \lambda(\omega t) d t=-(1 / 2) \cos \alpha$, which is the same as the stability in the averaged model. The state $\psi=0$ is thus linearly stable for $-\pi / 2<\alpha<\pi / 2$. However, it should be noted that $\lambda(\omega t)$ can be negative for some range of time even when its time average is positive. Hence it seems likely that the system is more easily destabilized in the nonaveraging model than the averaging one. Nevertheless, the reentrant transition is observed even when there is no time interval for the coefficient to be negative. In fact, considering the case $\alpha=0$, where $\lambda(\omega t)=\sin ^{2} \omega t \geqslant 0$, the synchronized state is never destabilized at any time, which suggests that nonlinearity is responsible for the reentrant transition.

To understand the global structure of the system, we again utilize a potential description. Equation (1) can be rewritten as

$$
\dot{\psi}=-K \frac{\partial F(\psi, t)}{\partial \psi}+\xi(t)
$$

where

$$
\begin{aligned}
F(\psi, t) \equiv & -\int_{0}^{\psi} d \psi^{\prime} Z\left(\psi^{\prime}+\omega t\right)\left\{h(\omega t)-h\left(\psi^{\prime}+\omega t\right)\right\} \\
= & -\frac{1}{2} \cos (\psi-\alpha)-\frac{1}{2} \cos (\psi+2 \omega t-\alpha) \\
& +\frac{1}{4} \cos (2 \psi+2 \omega t-\alpha)+\frac{1}{2}(\sin \alpha) \psi \\
& +\frac{1}{2} \cos \alpha+\frac{1}{4} \cos (2 \omega t-\alpha) .
\end{aligned}
$$

Note that the potential $F(\psi, t)$ is now time dependent. In general, $F(\psi, t)$ is a $2 \pi$-periodic function in $\omega t$. Choosing $Z$ and $h$ as in Eq. (2), $F$ is $\pi$-periodic in $\omega t$. Figure 2 shows the space-time plot of $F(\psi, t)$ for $\alpha=0$. It is clearly seen that, in addition to the minimum $\psi=0$, which exists in the case of the averaged dynamics as well, there is another minimum traveling in the negative direction of $\psi$. One can easily confirm that $F(\psi, t)$ has the traveling minimum $\psi=-2 \omega t$ and the two maxima $-\omega t+\alpha$ and $-\omega t+\alpha+\pi$. It is expected that, in the nonaveraged dynamics, phase slip occurs along this traveling minimum.

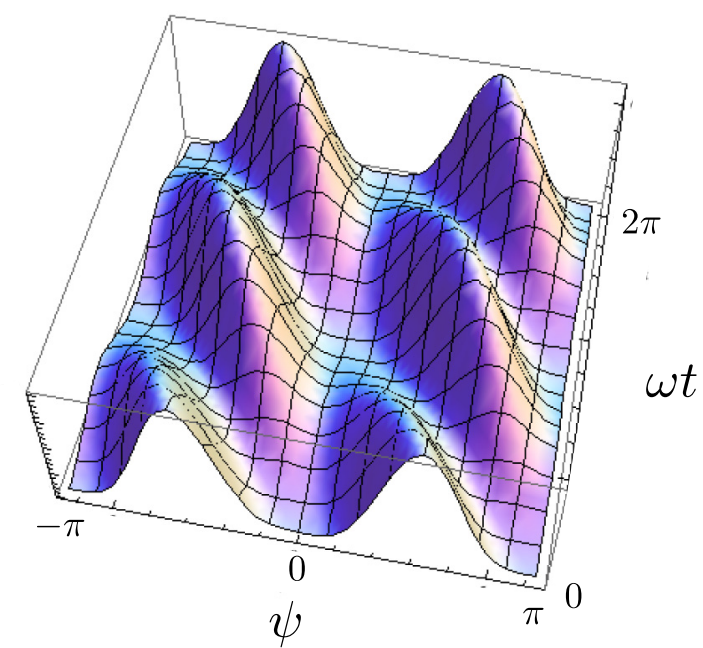

FIG. 2. (Color online) Space-time plot of the time dependent potential $F(\psi, t)$ [Eq. (12)] for $\alpha=0$.

The potential is not tilted if $\alpha=0$, and tilted if $\alpha \neq 0$. Since the former is easier to analyze, below we first present our analysis for $\alpha=0$, and then extend the analysis to the case $\alpha \neq 0$.

\section{Nontilted potential $(\alpha=0)$}

The mechanism and condition of the phase slip can be understood through the analysis of the following FokkerPlanck equation:

$$
\frac{\partial P(\psi, t)}{\partial t}=K \frac{\partial}{\partial \psi}\left(\frac{\partial F}{\partial \psi} P\right)+\frac{D}{2} \frac{\partial^{2} P}{\partial \psi^{2}},
$$

where $P(\psi, t)$ is the probability distribution function for the phase difference $\psi$. By numerically solving Eq. (13) with Eq. (12) for $\alpha=0$, we obtain time evolution of $P(\psi, t)$. Figure 3(a) shows $P(\psi, t)$ together with the trajectories of the extrema of $F(\psi, t)$. Note that, for $\alpha=0$, three of them cross each other at the same time. When the coupling is sufficiently large, $P$ splits into two components at some moment, one localized at $\psi=0$ corresponding to the synchronized state, and the other traveling along $\psi=-2 \omega t$, which corresponds to the phase slip state. This traveling component appears only for sufficiently strong coupling, although the distribution around $\psi=0$ is sharper for stronger coupling [Figs. 3(b) and 3(c)].

The scenario of how the traveling component emerges is as follows. Let us focus on a short time interval around $t=0$. When $t<0, P$ is localized at $\psi=0$. At $t=0$, the three extrema of $F$ cross each other [Fig. 3(a)]. Around this time, the curvature of the potential at $\psi=0$ almost vanishes, and hence the diffusion dominates the dynamics. Then, for $t>0$, a potential maximum located at $\psi=-\omega t$ gradually grows, and at some time $t_{c}$ the drift force caused by the potential becomes comparable to the effect of diffusion. At this moment, the part of $P(\psi, t)$ located beyond the maximum (i.e., $\psi<$ $-\omega t)$ is separated from the component around $\psi=0$ and thus conveyed with the potential minimum located at $\psi=-2 \omega t$. This process repeats itself with period $\pi$. 

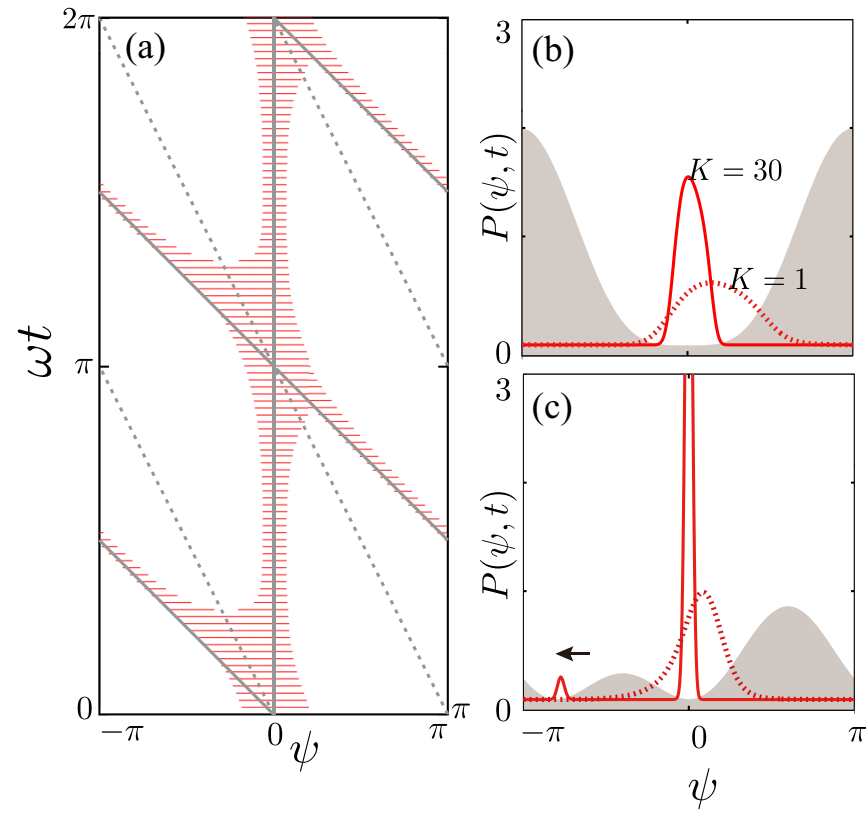

FIG. 3. (Color online) (a) Space-time plot of the probability distribution function $P(\psi, t)$ obtained by solving Eq. (13) with $K=30, D=0.1$, and $\alpha=0$. Only the region $P(\psi, t)>0.01$ is shown (red). The minima (solid lines) and the maxima (dotted lines) of the potential $F(\psi, t)$ are also shown. (b),(c) Snapshots of $P(\psi, t)$ for $K=1$ (dotted) and $K=30$ (solid), with $D=0.1$ and $\alpha=0$. Shaded regions represent $F(\psi, t)$. (b) $t=0$, (c) $t=1.25$. Probability current corresponding to the phase slip is indicated by an arrow in (c).

We can roughly estimate the above $t_{c}$ and the transition line through a dimensional analysis. First, $t_{c}$ is determined by balancing the drift and the diffusion terms in Eq. (13) evaluated at the potential maximum $\psi=-\omega t$ :

$$
K F\left(-\omega t_{c}, t_{c}\right) \sim D .
$$

Because diffusion is dominant for $0<t<t_{c}$, the width of $P$ grows roughly as $\sqrt{D t}$ within this duration. If this width is comparable to distance to the potential maximum at $t=t_{c}$, i.e.,

$$
\sqrt{D t_{c}} \sim \omega t_{c},
$$

a substantial part of $P$ will be conveyed. From Eqs. (14) and (15), and noting that the potential height for small $t$ is $F(-\omega t, t) \sim(\omega t)^{4}$, we obtain the following scaling relation:

$$
D \sim \omega^{4 / 3} K^{-1 / 3} \text {. }
$$

By substituting Eq. (16) into Eq. (15), we obtain the relationship between $K$ and $t_{c}$ :

$$
t_{c} \sim \omega^{-2 / 3} K^{-1 / 3}
$$

The reason why stronger coupling induces more phase slips is now clear: Since larger coupling strength $K$ implies smaller $t_{c}$, the condition $\sqrt{D t_{c}}>\omega t_{c}$ is easier to satisfy.

\section{Tilted potential $(\alpha \neq 0)$}

In the case of small but nonzero $|\alpha|$, where the three extrema cross each other at different timings, a similar argument

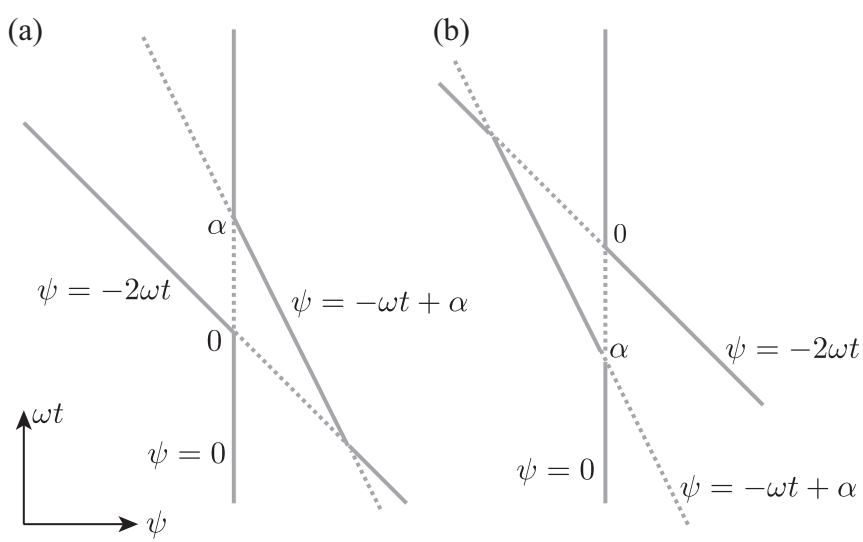

FIG. 4. Schematic of potential extrema crossing for nonzero $\alpha$ : (a) $\alpha>0$, (b) $\alpha<0$. Solid (dotted) lines correspond to minima (maxima) of the potential $F(\psi, t)$.

can still be made to obtain the transition lines: If $\alpha>0$, $\psi=0$ becomes unstable when $\psi=-2 \omega t$ crosses 0 at $t=0$. On the other hand, if $\alpha<0, \psi=0$ loses its stability at $t=-\omega^{-1}|\alpha|$, when $\psi=-\omega t+\alpha$ crosses $\psi=0$. In both cases, the potential barrier is located at $\psi=-\omega t+\alpha$. These situations are schematically shown in Fig. 4. Hence, instead of Eq. (15), we have different balance equations $\lambda \sqrt{D t_{c}}=$ $\omega t_{c}-\alpha(\alpha \geqslant 0)$ and $\lambda \sqrt{D\left(t_{c}-\omega^{-1} \alpha\right)}=\omega t_{c}-\alpha(\alpha<0)$, where a parameter $\lambda$ has been introduced. The transition line is thus obtained by eliminating $t_{c}$ from the following equations:

$$
\begin{gathered}
K F\left(-t_{c}+\alpha, t_{c}\right)=D, \\
\omega t_{c}= \begin{cases}\frac{\omega^{-1} D \lambda^{2}}{2}+\alpha+\sqrt{\left(\frac{\omega^{-1} D \lambda^{2}}{2}+\alpha\right)^{2}-\alpha^{2}} & (\alpha \geqslant 0), \\
\lambda^{2} \omega^{-1} D+\alpha & (\alpha<0) .\end{cases}
\end{gathered}
$$

Substituting $\alpha=0$ obviously reproduces Eqs. (14) and (15). Theoretical lines given by Eqs. (18) and (19) with $\lambda=3.0$ agree well with numerical data for $\alpha=0$ and $\alpha=0.3$ in Fig. 1(b), although for $\alpha=-0.3$ it slightly deviates from the numerical data particularly for large $K$. This result suggests that the sign of $\alpha$ is critical for stable synchronization: phase slip hardly occurs if $\alpha<0$ even for large $K$.

\section{Frequency mismatch}

We can further extend our scaling argument for $\alpha=0$ to the case $\Omega \neq \omega$. Since the frequency difference $\Delta \omega=\omega-\Omega$ serves as a drifting force, Eq. (15) is now modified as

$$
\lambda \sqrt{D t_{c}}-\sigma \Delta \omega t_{c}=\omega t_{c},
$$

where $\sigma>0$ is another parameter. Combining Eq. (20) with Eq. (14), we obtain

$$
D \sim \omega^{\frac{4}{3}}\left(1+\sigma \frac{\Delta \omega}{\omega}\right)^{8 / 3} K^{-1 / 3} .
$$

Figure 5(a) shows the phase slip rates for different values of $\Delta \omega$. Rescaling according to Eq. (21) results in the collapse of data points [Fig. 5(b)], which strongly supports the validity of our theory. 
(a)

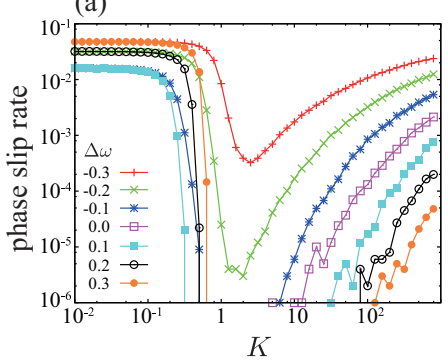

(b)

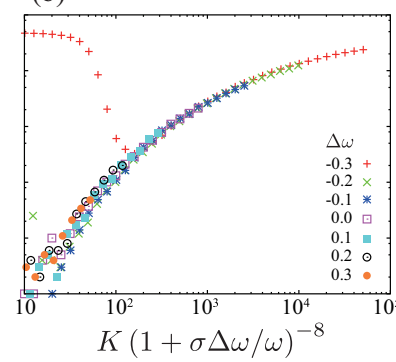

FIG. 5. (Color online) (a) Phase slip rates against $K$ for different $\omega=\Delta \omega+\Omega$, with $D=0.3, \alpha=0$, and $\Omega=1$. (b) Same data plot for larger $K$, scaled by $(1+\sigma \Delta \omega / \omega)^{8}$, with the fitting parameter $\sigma=1.35$.

\section{Generality of reentrant transition}

We have studied the nonaveraged phase model (1) using a specific form of $Z$ and $h$ as in Eq. (2). Now let us consider reentrant transition in more general settings. For simplicity, we again focus on the case $\Omega=\omega$. As we have observed above, the extrema of the potential $F(\psi, t)$, which are given by zeros of $Z(\psi+\omega t)$ and $H(\psi, t) \equiv h(\omega t)-h(\psi+\omega t)$ for $0 \leqslant \psi \leqslant 2 \pi$, play important roles on phase slip. Obviously $H$ has a zero at $\psi=0$, which corresponds to the synchronized state. Now we assume that the functions $Z$ and $h$ are unimodal and that $Z$ has two zeros, which are reasonable in many practical cases. Then it follows that $H$ has another zero $\psi=\beta(t)$, which we call the moving $H$ branch, corresponding to phase slip. In fact, from the above assumption it is easy to show that $\beta(t)$ is a monotonically decreasing function of $t$ that satisfies $\frac{d \beta(t)}{d t}<-\omega$, crossing $\psi=2 n \pi(n=0,1,2, \ldots)$ twice within one oscillation period, reflecting the fact that phase slips occur in the negative direction. In addition, two zeros of the response function $Z(\psi+\omega t)$, denoted by $\alpha_{1}$ and $\alpha_{2}$, give the other potential extrema moving linearly along $\psi=-\omega t+\alpha_{1,2}$, which we call $Z$ branches. Note that, in the case of Eq. (2), the $Z$ branches are $\psi=-\omega t+\alpha$ and $-\omega t+\alpha+\pi$, and the moving $H$ branch is $\psi=-2 \omega t$.

The synchronized state $\psi=0$ is destabilized by the crossing of the $Z$ branches and the moving $H$ branches, and phase slip may occur, depending on in which order these branches cross $\psi=0$ : if the branch crossing is like Fig. 4(a), that is, if $\psi=0$ is destabilized by the moving $H$ branch first, and then restabilized by a $Z$ branch, phase slip is more likely to occur; if the crossing is like Fig. 4(b), phase slip is less likely.

\section{REENTRANT TRANSITION IN COUPLED LIMIT CYCLE OSCILLATORS}

Let us confirm that the reentrant transition occurs in limit cycle oscillators. We demonstrate it by using the Brusselator model:

$$
\begin{gathered}
\dot{u}_{i}=A-(B+1) u_{i}+u_{i}^{2} v_{i}+K_{i}^{(u)}\left(u_{j}-u_{i}\right)+\xi_{i}(t), \\
\dot{v}_{i}=B u_{i}-u_{i}^{2} v_{i}+K_{i}^{(v)}\left(v_{j}-v_{i}\right)+\eta_{i}(t),
\end{gathered}
$$

where $(i, j)=(1,2)$ or $(2,1), u_{i, j}$ and $v_{i, j}$ are the state variables, and $\xi_{i}(t)$ and $\eta_{i}(t)$ are the Gaussian white noise satisfying $\left\langle\xi_{i}(t) \xi_{i}\left(t^{\prime}\right)\right\rangle=\left\langle\eta_{i}(t) \eta_{i}\left(t^{\prime}\right)\right\rangle=D_{i} \delta\left(t-t^{\prime}\right)$. Here we consider
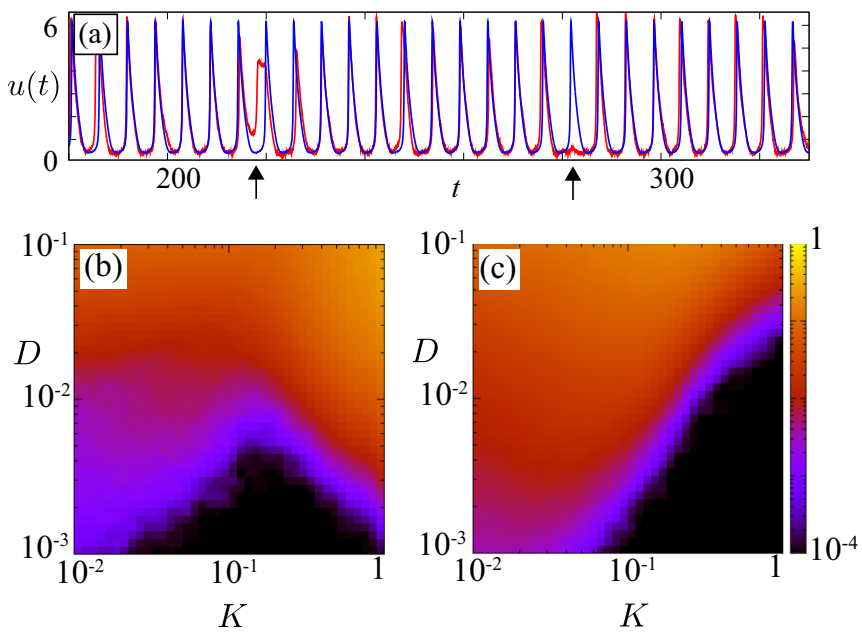

FIG. 6. (Color online) Two unidirectionally coupled Brusselators: Parameters are $A=1.6$ and $B=5.0$. (a) Time series of $u_{1}(t)$ (red) and $u_{2}(t)$ (blue) in the case of $v$ coupling, with $K=0.5$ and $D=0.01$. Oscillation failures are observed at $t \sim 220$ and $t \sim 280$, indicated by arrows. (b),(c) Frequency of phase slip against $D$ and $K$ : (b) $v$ coupling, (c) $u$ coupling.

the case where oscillator 2 acts as a noise-free pacemaker: $D_{2}=0, K_{2}^{(u)}=K_{2}^{(v)}=0$. On the other hand, oscillator 1 is under noise, $D_{1}=D$, and is influenced by oscillator 2 via $u$ coupling $\left(K_{1}^{(u)}=K, K_{1}^{(v)}=0\right)$ or $v$ coupling $\left(K_{1}^{(u)}=0\right.$, $\left.K_{1}^{(v)}=K\right)$.

In the case of $v$ coupling, too strong coupling results in occasional oscillation failures, as shown in Fig. 6(a). If we measure the phase of the state $(u, v)$ in a standard way [2], oscillation failure can be expressed as phase slip. The frequency of phase slips increases as $K$ increases for fixed $D$, indicating a reentrant transition from synchronization to phase slip [Fig. 6(b)]. On the other hand, $u$ coupling does not induce the reentrant transition [Fig. 6(c)].

The difference between the two ways of coupling can be understood by applying the analysis in Sec. II C to the phase model of the Brusselator. We numerically solve Eq. (1) with $Z$ and $h$ calculated from the numerical phase reduction of Eqs. (22) and (23). The trajectory of the phase difference $\psi(t)$ and the potential extrema are shown in Fig. 7. Here we can clearly see the two types of branch crossing discussed in Sec. II C: In the case of $v$ coupling [Fig. 7(a)], it is observed that $\psi(t)$ occasionally leaves the state $\psi=0$ and travels along the moving $H$ branch. This occurs when the state $\psi=0$ becomes temporally unstable due to the crossing by the moving $H$ branch. Its stability recovers when the state $\psi=0$ is crossed by one of the $Z$ branches. This branch crossing is the type $\alpha>0$ [Fig. 4(a)]. On the other hand, in the case of $u$ coupling, phase slip is not observed [Fig. 7(b)], where branch crossing is of the type $\alpha<0$ [Fig. 4(b)]. These results correspond to the presence and the absence of the reentrant transition in Figs. 6(b) and 6(c), respectively.

It is remarkable that the reentrant transition in the Brusselator model is well understood from the analysis of its corresponding phase model for such a strong coupling case. Our results indicate that nonaveraged phase models are useful for the understanding of strongly coupled oscillators. 

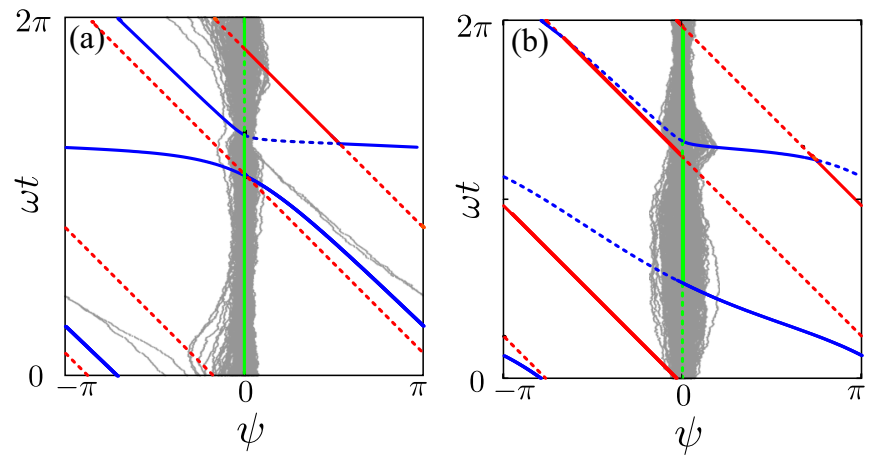

FIG. 7. (Color online) Trajectories (gray) of the phase difference $\psi(t)$ obtained by solving Eq. (1), with $Z$ and $h$ calculated from the numerical phase reduction of Eqs. (22) and (23), with $D=0.005$, $K=1.0$. The extrema of the numerically constructed potential $F(\psi, t)$ are shown together: $\psi=0$ (green), $Z$ branches (red), and a moving $H$ branch (blue). Solid and dotted lines correspond to minima and maxima of the potential, respectively. The trajectory is plotted modulo $2 \pi$ in $\omega t$. (a) $v$ coupling, (b) $u$ coupling.

\section{CONCLUDING REMARKS}

Our study has uncovered that, in addition to a lower limit in coupling strength, there is generally an upper limit over which synchronization is disrupted by phase slips when an oscillator is subject to noise. Therefore, when strong coupling is required, the way of coupling should carefully be constructed.

In this study we have only considered unidirectional coupling. In the case of mutual coupling, we have confirmed in a preliminary numerical study that mutual coupling also exhibits reentrant transition, although oscillation death is more commonly observed.

A similar reentrant transition is known to occur in a certain class of chaotic oscillators [19,20]. Our study would further motivate such studies.

\section{ACKNOWLEDGMENT}

We thank Dr. F. Mori for valuable discussions.
[1] A. T. Winfree, The Geometry of Biological Time, 2nd ed. (Springer, New York, 2001).

[2] Y. Kuramoto, Chemical Oscillations, Waves, and Turbulence (Springer, New York, 1984).

[3] A. Pikovsky, M. Rosenblum, and J. Kurths, Synchronization: A Universal Concept in Nonlinear Sciences (Cambridge University Press, Cambridge, UK, 2001).

[4] L. H. van der Tweel, F. L. Meijler, and F. J. L. van Capelle, J. Appl. Physiol. 34, 283 (1973).

[5] L. Glass, Nature (London) 410, 277 (2001).

[6] S. M. Reppert and D. R. Weaver, Nature (London) 418, 935 (2002).

[7] Y. Yamaguchi et al., Science 342, 85 (2013).

[8] A. E. Motter, S. A. Myers, M. Anghel, and T. Nishikawa, Nat. Phys. 9, 191 (2013).

[9] F. Dörfler, M. Chertkov, and F. Bullo, Proc. Natl. Acad. Sci. USA 110, 2005 (2013).

[10] M. Rohden, A. Sorge, M. Timme, and D. Witthaut, Phys. Rev. Lett. 109, 064101 (2012).
[11] J. T. Ariaratnam and S. H. Strogatz, Phys. Rev. Lett. 86, 4278 (2001).

[12] G. B. Ermentrout, Physica D 41, 219 (1990).

[13] U. Parlitz and W. Lauterborn, Phys. Rev. A 36, 1428 (1987).

[14] K. Ito and Y. Nishiura, Phys. Rev. E 77, 036224 (2008).

[15] D. García-Álvarez, A. Bahraminasab, A. Stefanovska, and P. V. E. McClintock, Europhys. Lett. 88, 30005 (2009).

[16] M. Komarov, S. Gupta, and A. Pikovsky, Europhys. Lett. 106, 40003 (2014).

[17] S. Gupta, A. Campa, and S. Ruffo, J. Stat. Mech: Theory Exp. (2014) R08001.

[18] H. Sakaguchi and Y. Kuramoto, Prog. Theor. Phys. 76, 576 (1986).

[19] J. F. Heagy, T. L. Carroll, and L. M. Pecora, Phys. Rev. E 50, 1874 (1994).

[20] L. Huang, Q. Chen, Y.-C. Lai, and L. M. Pecora, Phys. Rev. E 80, 036204 (2009). 